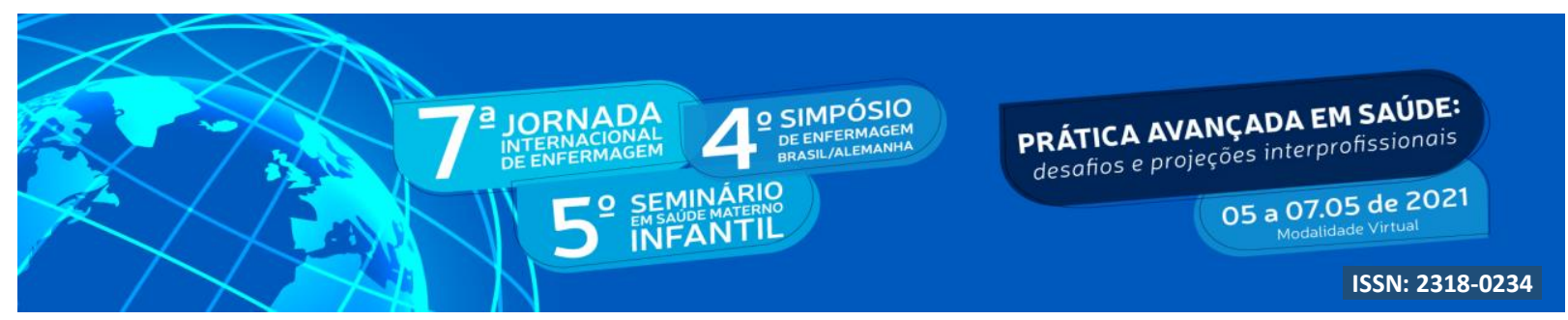

DOI: http://doi.org/10.48195/jie2021-146

\title{
AURICULOTERAPIA E ANSIEDADE: EVIDÊNCIAS NA LITERATURA ${ }^{1}$
}

\section{Marina Valcanover Borges ${ }^{2}$; Jucimara Montagner Michelon³ ${ }^{3}$ Márcio Rossato Badke 4 , Marciele Pinheiro ${ }^{5}$; Fabiana Porto da Silva ${ }^{6}$; Rosiane Filipin Rangel ${ }^{7}$}

\section{RESUMO}

Objetivo: Investigar as evidências na literatura sobre os efeitos da auriculoterapia na ansiedade. Método: Revisão Narrativa de Literatura, realizada entre os meses de agosto a setembro de 2020, nas bases de dados Literatura Latino-Americana e do Caribe em Ciências da Saúde, Medical Literature Analysis and Retrieval System Online e na Biblioteca Eletrônica Científica Online. Resultados: foi possível a construção de duas categorias sobre o uso da auriculoterapia na redução da ansiedade, quais sejam: ansiedade e o ciclo gravídico: Benefícios da auriculoterapia e redução dos níveis de ansiedade por meio da auriculoterapia. Considerações finais: Os estudos encontrados evidenciaram resultados significativos na redução da ansiedade com o uso da auriculoterapia. Quanto às limitações da pesquisa conclui-se que foram o pequeno número de estudos encontrados, no entanto, por ser uma revisão narrativa da literatura sugerem-se mais estudos com novas populações e em diferentes contextos para que se avance nesse conhecimento.

Palavras-chave: Terapias Complementares; Auriculoterapia; Ansiedade; Enfermagem.

\begin{abstract}
Objective: To investigate literature evidence on the effects of auriculotherapy on anxiety. Method: Narrative Literature Review, carried out between August and September 2020, in the Latin American and Caribbean Literature in Health Sciences, Medical Literature Analysis and Retrieval System Online and the Library databases Online Scientific Electronics. Results: Two categories were found on the use of auriculotherapy to reduce anxiety, namely: "Anxiety and the pregnancy cycle: Benefits of auriculotherapy" and "Reduction of anxiety levels through auriculotherapy". Final considerations: It is can be said that objective of this research was achieved. Because it was found that the studies analyzed here present significant results in reducing anxiety symptom with the use of therapy. The limitations of this study consists in the small number of studies found in the previous literature, however, as it is a narrative review of the literature, further studies with new populations and in different contexts are suggested, so that the field can advance on this topic.
\end{abstract}

Key Words: Complementary Therapies; Auriculotherapy; Anxiety; Nursing.

\footnotetext{
${ }^{1}$ Trabalho de pesquisa.

${ }^{2}$ Enfermeira. Especializanda em Oncologia e Hematologia pela UNINTER. Hospital Santa Cruz, Santa Cruz RS. E-mail: marina.valcanover@hotmail.com

${ }^{3}$ Enfermeira Obstetra. Aluna do Mestrado Profissional em Saúde Materno Infantil da Universidade Franciscana - UFN. Hospital Casa de Saúde. Santa Maria, RS. E-mail: enfer.jucimara@ gmail.com

${ }^{4}$ Enfermeiro. Doutor em Ciências pela Universidade Federal de Pelotas - UFPEL. Professor Adjunto da Universidade Federal de Santa Maria - UFSM. Santa Maria, RS. E-mail: marciobadke@gmail.com

${ }^{5}$ Enfermeira. Mestre em Saúde Materno Infantil pela Universidade Franciscana - UFN. Hospital Universitário de Santa Maria - HUSM. Santa Maria, RS. E-mail: marcielepinheiro@hotmail.com.br

${ }^{6}$ Enfermeira. Mestre em Ciências da Saúde e da Vida pela Universidade Franciscana - UFN. Docente da Universidade Franciscana - UFN. E-mail: fabiana.silva@ufn.edu.br

7 Orientadora. Enfermeira. Doutora em Enfermagem pela Universidade Federal do Rio Grande - FURG. Docente da Universidade Franciscana - UFN. Santa Maria, RS. E-mail: rosiane@ufn.edu.br
} 


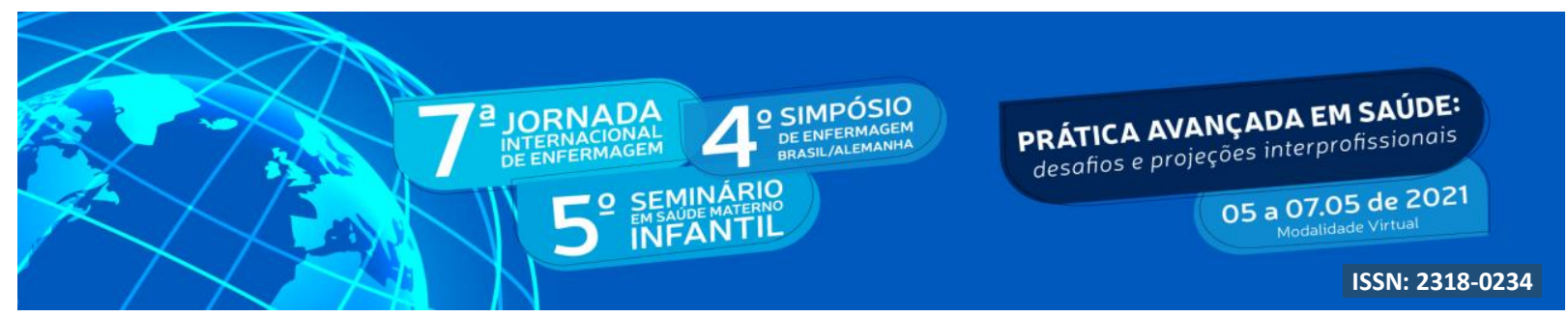

\section{INTRODUÇÃO}

De acordo com a Organização Mundial de Saúde (OMS), no Brasil estão os maiores indicadores de Transtorno de Ansiedade (TA), em média 9,3\% da população, enquanto a prevalência mundial está em 3,6\% (OMS, 2017). A ansiedade comumente se intensifica no contexto das pressões, demandas e estresses da vida diária, sendo uma reação natural e adaptativa. Pode ser definida como uma emoção orientada ao futuro sobre eventos potencialmente aversivos e/ou perigosos, que gera reações fisiológicas, comportamentais e afetivas que mobilizam o indivíduo para se preparar para possíveis ameaças. No entanto, pode tornar-se um transtorno quando: (1) é baseada em uma suposição falsa ou raciocínio falho sobre o potencial para ameaça ou perigo em situações relevantes; (2) quando interfere na capacidade do indivíduo para enfrentar as circunstâncias aversivas ou difíceis; (3) quando está presente por um período de tempo prolongado (SCHONHOFEN et al., 2020).

No panorama atual a sobrecarga de trabalho, consumismo, excesso de atividades e competitividade interferem significativamente na manifestação dos sinais e sintomas de ansiedade (GUIMARÃES et al., 2015). Nesse ínterim, buscando possibilidades que auxiliem as pessoas no cuidado à sua saúde na multidimensionalidade, destacam-se as Práticas Integrativas e Complementares (PICs).

Dentre as terapias, destaca-se a auriculoterapia, ou acupuntura auricular, essas, são conhecidas também por Medicina Tradicional Chinesa (MTC). A referida técnica usa a estimulação de pontos localizados no pavilhão auricular para efetuar tratamentos de enfermidades físicas e mentais devido ao reflexo que a orelha, por meio de seus inúmeros filetes nervosos e vasos capilares, exerce no Sistema Nervoso Central (SNC), e através deste, sobre o organismo. É considerada assim um método completo de terapia (SOUZA, 2012), que pode ser coadjuvante ao tratamento principal melhorando a resposta do mesmo, ou até mesmo um tratamento prioritário, ao longo do tempo, a fim de prolongar o efeito da terapêutica na pessoa (MOURA et al., 2015).

Estudo realizado por Moura et al. (2015) utilizando a auriculoterapia evidenciou que esta pode ser benéfica para a redução da ansiedade, sendo considerada um tratamento seguro, de rápida aplicação, realizado em diversas condições locais e ambientais, na expectativa de 


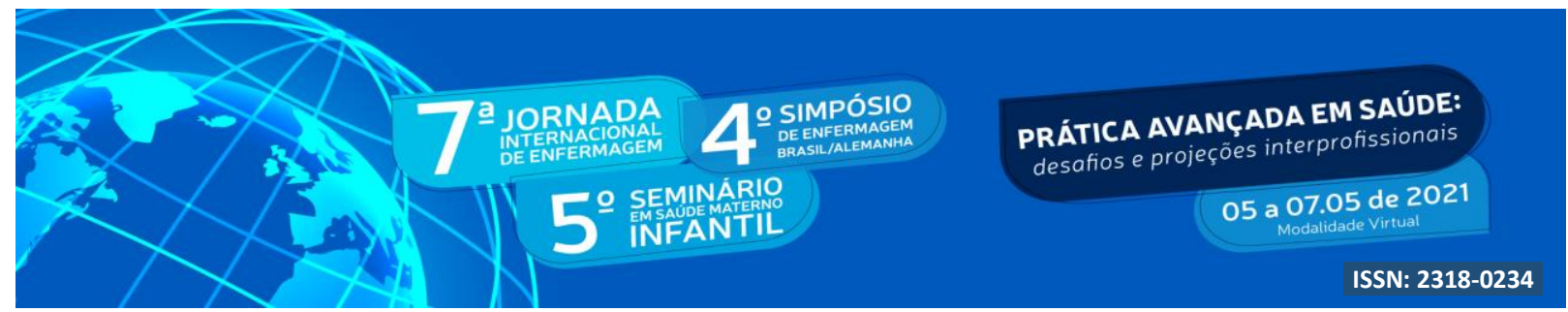

melhorar a qualidade de vida da população, uma vez que pode contribuir para reduzir, entre outras condições, a frequência cardíaca, a dor e a ansiedade.

Diante do que foi exposto, formulou-se a questão pesquisa do estudo: quais as evidências na literatura sobre os efeitos da auriculoterapia na ansiedade?

\section{OBJETIVO}

Investigar as evidências na literatura sobre os efeitos da auriculoterapia na ansiedade.

\section{METODOLOGIA}

Trata-se de uma Revisão Narrativa da Literatura (RNL). A busca do material foi realizada entre os meses de agosto a setembro de 2020, nas bases de dados Literatura LatinoAmericana e do Caribe em Ciências da Saúde (LILACS), Medical Literature Analysis and Retrieval System Online (MEDLINE) e na Biblioteca Eletrônica Científica Online (SCIELO), por meio dos descritores "Auriculoterapia" and "Ansiedade". Não foi estabelecido recorte temporal, visando obter um maior número de publicações.

Utilizou-se como critérios de inclusão artigos na íntegra, nos idiomas português e espanhol, disponíveis gratuitamente nas bases e, de exclusão: teses, dissertações, manuais técnicos, capítulos de livros e artigos que não contemplavam a temática de acordo com o objetivo proposto e estivessem repetidos nas bases.

Inicialmente, obteve-se cinco produções no LILACS, 27 no MEDLINE e sete no SCIELO, totalizando 39. Na etapa seguinte, ao aplicar os critérios de inclusão e exclusão, bem como fazer a leitura na íntegra dos estudos, finalizou-se a amostra em oito artigos. $\mathrm{Na}$ sequência, procedeu-se uma leitura, a fim de identificar os elementos explícitos e implícitos acerca do tema. Os resultados foram agrupados e categorizados para melhor visualização.

\section{RESULTADOS E DISCUSSÃO}

A disposição dos artigos ocorreu no período de 2012 a 2020, sendo (2) publicados em 2020, (1) em 2019, (2) em 2018, (1) em 2017, (1) em 2014 e (1) em 2012, observando-se um aumento nos últimos três anos. Notou-se uma diversificação na população estudada, pois dos 


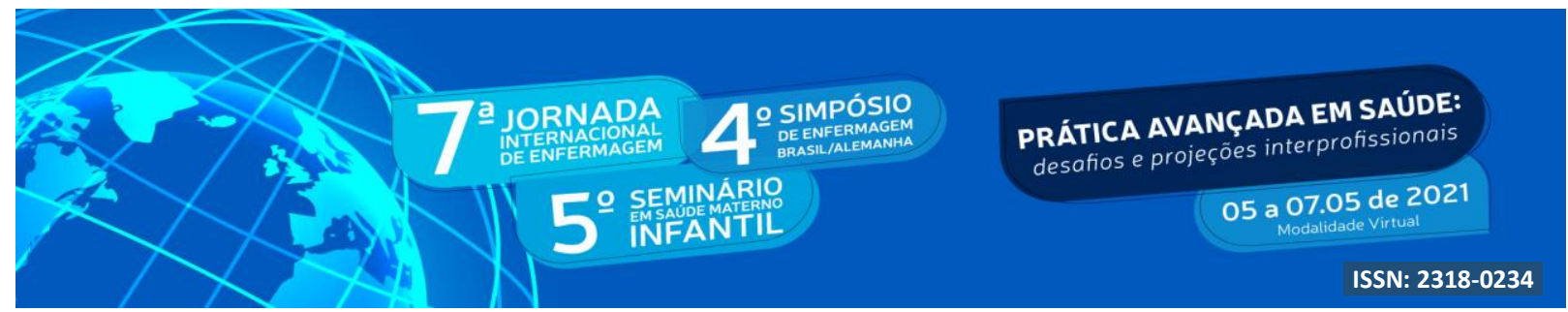

oito artigos: (3) foram realizados com gestantes, (1) com profissionais do sistema penitenciário, (1) com profissionais de enfermagem, (1) com estudantes de enfermagem, (1) com pessoas desnutridas e (1) pesquisa bibliográfica.

As populações estudadas podem ter sido escolhidas por apresentarem vulnerabilidade a condições que proporcionam ansiedade. Nesse contexto, Jongsma et. al. (2018) revela que homens, jovens, minorias étnicas e moradores de áreas com baixos indicadores socioeconômicos têm maior propensão a apresentar um primeiro episódio psicótico. Esses dados indicam que a ansiedade é um fenômeno que acomete as mais diversas populações e necessita de mais estudos que contemplem as especificidades de cada população.

Os autores dos artigos analisados empregaram variados métodos de estudo para apoiar suas pesquisas tais como: (1) estudo experimental, (2) revisão integrativa, (2) estudo clínico randomizado simples-cego, (1) ensaio clínico randomizado, paralelo e triplo-cego, (1) ensaio clínico randomizado e (1) estudo quase experimental. Ainda, referente ao idioma de publicação, (6) artigos foram publicados em português e (2) em espanhol.

As publicações estão distribuídas em (5) periódicos da área da Enfermagem, sendo (2) na Revista Latino-Americana de Enfermagem, (1) Revista Escola de Enfermagem USP, (1) Revista Cubana de Enfermería e (2) Escola Paulista de Enfermagem, (1) Universidad y Salud e (1) Sociedade Brasileira do Estudo da Dor. Esses dados demonstram a predominância de publicações vinculadas a revistas de enfermagem.

Através da leitura dos estudos, foram encontradas duas categorias sobre o uso da auriculoterapia na redução da ansiedade. A seguir, serão abordados os principais tópicos elencados.

\section{Ansiedade e o ciclo gravídico: Benefícios da auriculoterapia}

Uma assistência humanizada à gestante abarca diversas ações, dentre elas o respeito aos direitos e escolhas das mulheres, a valorização do conhecimento popular e as possibilidades terapêuticas que podem ser utilizadas concomitantemente ao modelo convencional de cuidado (SILVA et al., 2016). Nessa direção, a utilização de PICs na gestação vem sendo abordada em diversos estudos como auxiliar na redução de sintomas indesejados que podem ser manifestados nesse período, bem como para o preparo no trabalho de parto. 


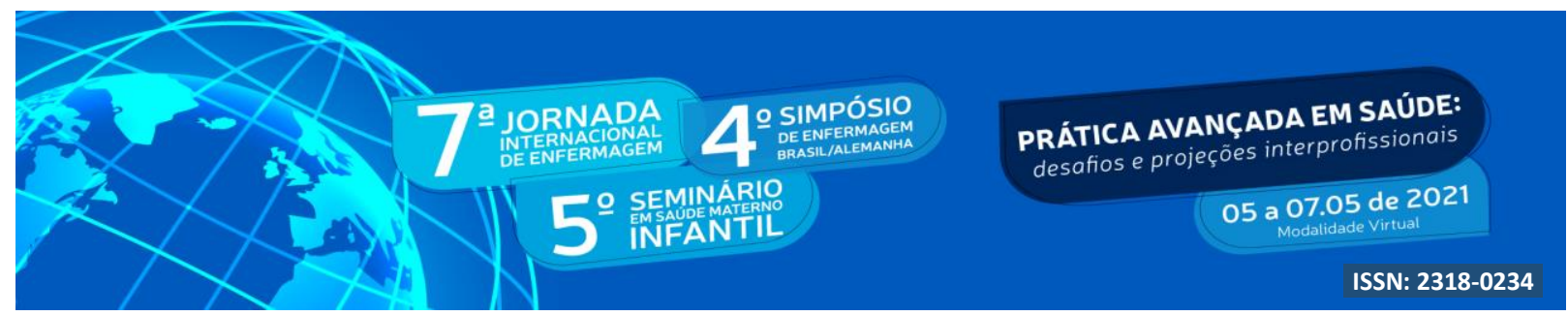

Dentre essas terapias, destaca-se a auriculoterapia, visto seu potencial no âmbito do SUS, bem como os resultados apresentados nas pesquisas (SILVA et al.,2020; MASCARENHAS et al., 2019).

Frente a isso, destaca-se estudo realizado com o objetivo de avaliar os efeitos da auriculoterapia nos níveis de ansiedade em gestantes atendidas em pré-natal de baixo risco. Os resultados evidenciaram que essa prática é uma alternativa eficaz quando utilizada como intervenção no que tange a redução dos níveis de ansiedade nas gestantes que são atendidas em pré-natal de baixo risco (SILVA et al., 2020).

Cabe salientar que a ansiedade, no período gravídico, é um achado frequente devido às alterações no cotidiano de vida dessas mulheres que é marcado por alterações hormonais, mudanças sociais, entre outros fatores que influenciam de maneira expressiva nas suas múltiplas dimensões (ARAÚJO et. al., 2016). Corroborando, Mafetoni, et al., (2018), esse sentimento torna-se mais expressivo sobretudo quando incide sobre parturientes com pouca instrução, primigestas e ambientes hospitalares com elevados índices de intervenções médicas.

Estudos evidenciam que a ansiedade é um dos fatores significativos que leva as mulheres a optarem pela cesariana (MASCARENHAS et al., 2019). Frente a isso, compreende-se a importância da utilização de métodos não farmacológicos que possibilitem minimizar esses sentimentos despertados durante a gestação e direcione as mulheres para decisões/escolhas que possibilitem o desencadear da fisiologia do parto, bem como as boas práticas obstétricas e neonatais.

Nessa direção, na pesquisa realizada por Mafetoni et al. (2018) em que objetivou-se avaliar a efetividade da auriculoterapia sobre a ansiedade de mulheres durante o trabalho de parto, os autores evidenciaram que as parturientes tratadas com essa terapia apresentaram menor nível de ansiedade quando comparadas às mulheres dos outros grupos. Outro estudo, desenvolvido por Cherobin; Oliveira; Brisola, (2016), utilizando acupuntura e a auriculoterapia, envolvendo a participação consentida de 19 parturientes admitidas em trabalho de parto no centro obstétrico de uma maternidade pública no estado de Santa Catarina, evidenciou que nos primeiros 30 minutos, quinze (79\%) das mulheres tratadas obtiveram algum alívio. Após $1 \mathrm{~h}$, seis (46\%) permaneceram com o mesmo grau de dor e 


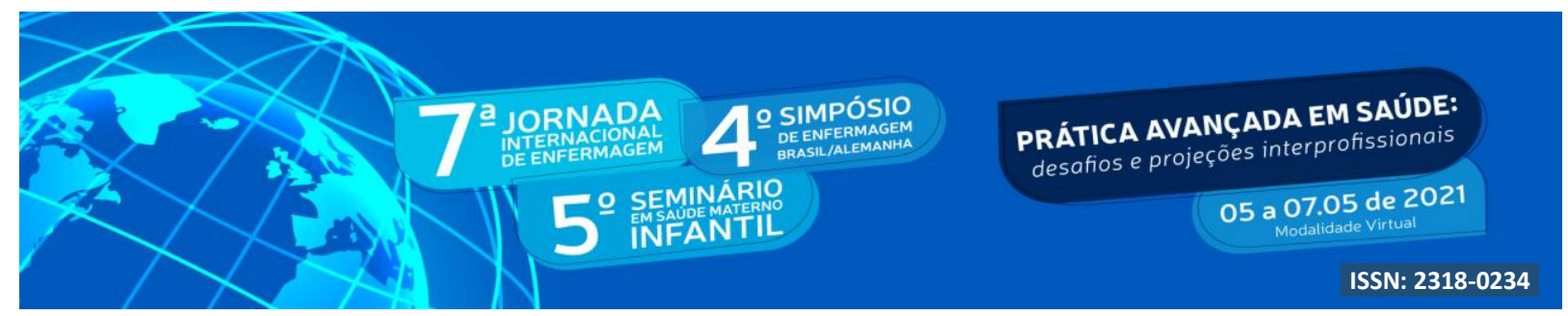

quatro $(31 \%)$ obtiveram um alívio maior.

A partir disso, compreende-se que os métodos não farmacológicos são importantes alternativas para alívio da dor e podem substituir/complementar a analgesia e métodos invasivos durante o trabalho de parto, contribuindo para um parto normal humanizado.

Destaca-se que as mulheres grávidas tendem a buscar as PICs durante esse período por diversas razões que incluem desde crenças até melhores experiências que não utilizem tantas intervenções farmacológicas e da medicina tradicional. No entanto, os estudos mostram que a maior parte das pacientes não revela o uso dessas práticas para o profissional de saúde (HALL; GRIFFITHS; MCKENNA, 2011). Esse achado direciona para uma reflexão acerca das práticas profissionais que ainda estão pautadas no modelo biomédico de cuidado que desconsidera, por vezes, o saber na sua complexidade, multidimensionalidade e integralidade.

\section{Redução dos níveis de ansiedade por meio da auriculoterapia}

Os transtornos de ansiedade têm aumentado expressivamente no último século, sobretudo devido às profundas transformações ocorridas no âmbito econômico e cultural que foram acompanhadas por pressões de uma sociedade moderna, tecnológica e, principalmente, cada vez mais competitiva (MARCHI et al., 2013).

Nessa direção, diversas são as alternativas para minimizar esse sentimento, dentre essas a auriculoterapia. Uma revisão da literatura que objetivou evidenciar sobre o efeito da auriculoterapia na ansiedade, concluiu que dos estudos analisados 78,11\% obtiveram redução desses níveis quando se utilizou essa terapia. Os autores pontuaram que as condições clínicas em que mais buscou-se a prática foram procedimentos de emergência, cirúrgicos, odontológicos e de pessoas que faziam uso de substâncias ilícitas (MOURA et al., 2015). Corroborando, um estudo desenvolvido por Santiago et al. (2018) com 21 adultos, entre 18 e 65 anos, de ambos os sexos, observou-se redução dos níveis de ansiedade após sete semanas de aplicação da auriculoterapia.

Convém salientar, que no contexto das universidades, essa realidade também tem sido evidenciada. Estudo demonstra que, ao ingressarem nesses cenários de formação, os estudantes são submetidos a uma grande carga de estresse, principalmente devido a cobranças pessoais, expectativas, familiares, encargos financeiros, saída de casa para morar em outra cidade, atividades domésticas, horários desordenados de sono e longas horas de estudo. Além 


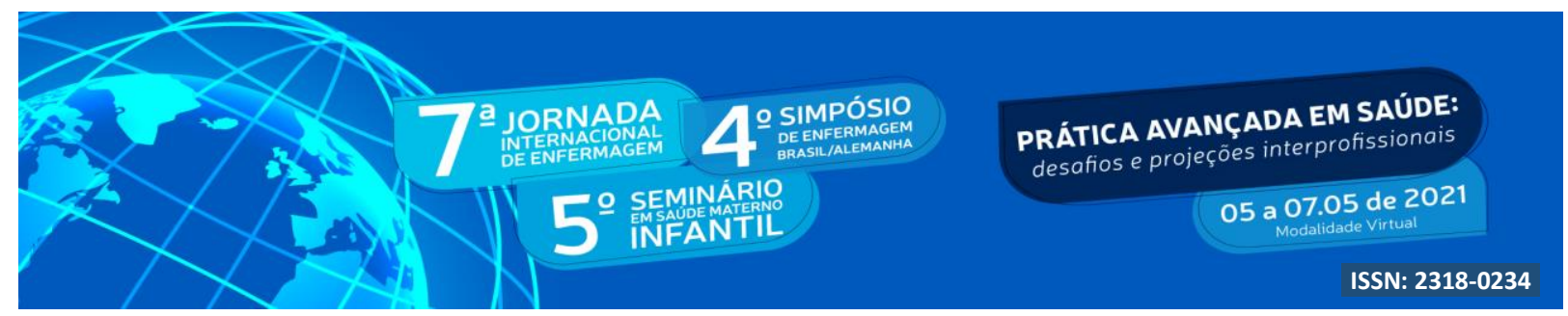

disso, transformações maturacionais (fisiológicas, neurológicas e psicológicas) decorrentes da transição entre a fase de adolescente e a de adulto, levam os mesmos a vivenciarem um período de crise, por exigir a adaptação a um novo papel social (MARCHI et al., 2013).

Nessa direção, compreende-se que os estudantes do ensino superior, precisamente os da área da saúde, formam um grupo que merece especial atenção, pois de acordo com as pesquisas, os mesmos apresentam maior nível de ansiedade, quando comparados a outras áreas de ensino. Esses, incluindo os estudantes de enfermagem, predispõem durante o período de graduação alguns fatores que podem ser desencadeadores de ansiedade. Destaca-se a experiência da prática clínica, o contato direto com o ser humano em situação de doença, por vezes em sofrimento psíquico, a observação constante dos instrutores no cenário da prática, o medo de cometer erros e sentimentos de inadequação, como os principais fatores que podem gerar sintomas ansiosos (LEÃO et al., 2018).

Também salienta-se que estes estudantes da área da saúde convivem muitas vezes com a situação da iminência de morte. Tais situações podem ser fonte de estresse e se tornarem exaustivas desencadeando conflitos internos e a exigência de um esforço de ajustamento e adaptação maior, surgindo, então, a necessidade de traçar estratégias que possibilitem a superação dessa situação (MARCHI et al, 2013).

Outra pesquisa realizada por Prado; Kurebayashi; Silva, (2012) com 71 acadêmicos de enfermagem demonstrou que a terapia realizada nos pontos Shenmen e Tronco cerebral foi mais eficaz para a diminuição dos níveis de ansiedade em comparação ao ponto Sham, ou seja, o placebo na pesquisa.

Além dos dados apresentados acima, os estudos com auriculoterapia também se mostram eficazes no tratamento da dor. Estudo realizado por Graça et al. (2020), desenvolvido na cadeia de um município da região médio-norte de Mato Grosso, incluindo 18 dos 22 profissionais que atuam na instituição, constituindo um grupo controle e um grupo intervenção, com nove participantes em cada e com objetivo de identificar como a auriculoterapia pode promover a qualidade de vida de profissionais do sistema penitenciário, mostrou resultados positivos no grupo intervenção, especialmente em relação ao estresse e lombalgia, o que aponta a efetividade da auriculoterapia nesse grupo, podendo trazer benefícios diretos aos profissionais, tanto em relação à disposição para o exercício laboral 


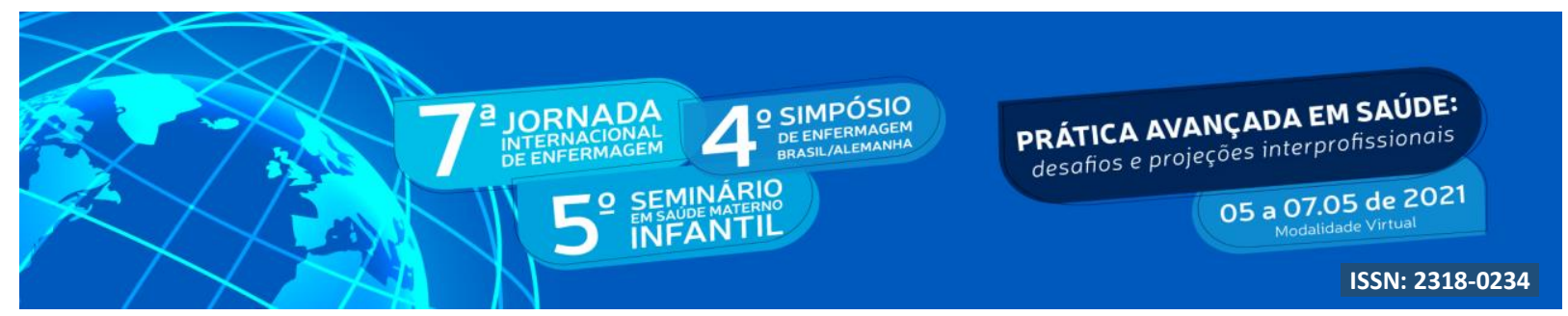

quanto para a promoção da qualidade de vida.

Em outra pesquisa realizada por Kurebayashi et al. (2017), com a finalidade de avaliar a efetividade do protocolo auricular para redução de ansiedade, dor (The Auricular Protocol for Pain \& Anxiety - APPA) e melhoria de qualidade de vida na equipe de Enfermagem de um hospital, foi proposto o tratamento como uma prática complementar e preventiva. Posto isso, com amostra inicial de 180 profissionais divididos em 4 grupos controle (G1), semente (G2), agulha (G3) e fita adesiva (G4) e utilizando os instrumentos de avaliação Inventário de Ansiedade Traço-Estado, Escala Visual Analógica de dor e instrumento de Qualidade de Vida, aplicados no início, depois de cinco e 10 sessões (cinco semanas), houve redução de $36 \%$ no G3, 24\% no G2 para a dor e $13 \%$ de aumento no nível mental de qualidade de vida para o G3, embora sem diferenças estatísticas. Logo, o protocolo APPA reduziu os níveis de ansiedade em equipe de enfermagem depois de 10 sessões.

A partir disso, entende-se que os estudos foram unânimes em apresentar os benefícios obtidos na promoção da saúde por meio da auriculoterapia. Contudo, todos eles evidenciam a necessidade de outras pesquisas que abordem a temática com um número maior de participantes no intuito de reforçar e aprofundar o conhecimento fazendo com que as PICs sejam efetivadas no cotidiano de prática dos profissionais da saúde e que a população possa ser beneficiada ainda mais desse conhecimento.

\section{CONCLUSÃO}

Os estudos encontrados evidenciaram resultados significativos na redução deste sintoma com o uso da terapia. Observou-se também a diversidade de estudos em que a técnica foi adotada, mostrando que pode ser utilizada em diversas situações e que é uma possibilidade terapêutica no cuidado profissional para promover a racionalização das ações de saúde, estimulando alternativas inovadoras e socialmente contributivas ao desenvolvimento sustentável de comunidades.

Sabe-se que a ansiedade é um fator que contribui de maneira negativa na qualidade de vida das pessoas, sendo assim, considera-se importante identificar os níveis de ansiedade e, a partir disso, possibilitar maneiras de cuidado que visem à integralidade humana, ou seja, que as dimensões física, psicológica, social e espiritual sejam atendidas. 


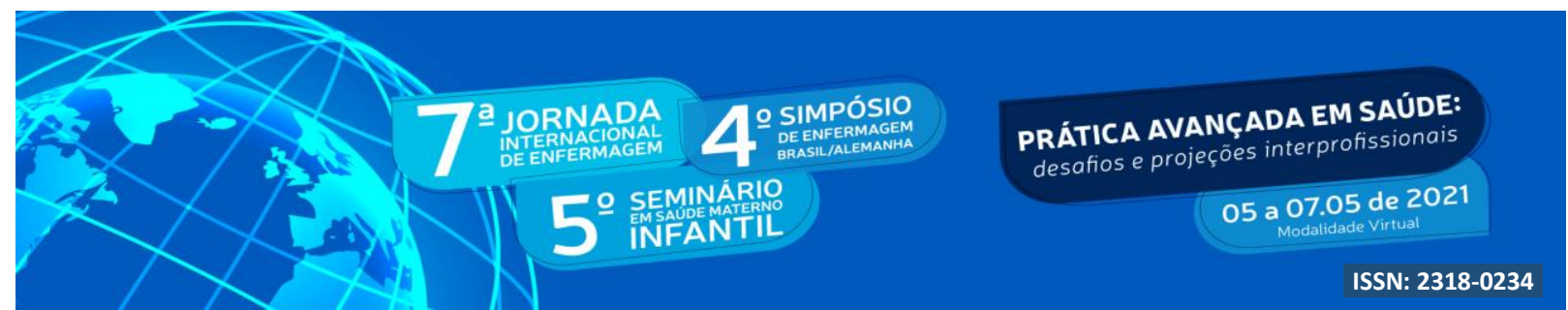

Quanto às limitações da pesquisa, conclui-se que foi o pequeno número de estudos encontrados na literatura, no entanto, por ser uma revisão narrativa sugerem-se mais investigações com novas populações e em diferentes contextos para que se avance nesse conhecimento.

\section{REFERÊNCIAS}

ARAÚJO, W.S. et al. Effects of relaxation on depression levels in women with high-risk pregnancies: a randomised clinical trial. Rev. Latino-Am. Enfermagem, Ribeirão Preto, v. 24, n. 2806, p: 1-8. 2016 .Disponível em: https://doi.org/10.1590/1518-8345.1249.2806. Acesso em; Nov. 2020.

CHEROBIN, F.; OLIVEIRA, A.R.; BRISOLA, A.M. Acupuntura e auriculoterapia como métodos não farmacológicos de alívio da dor no processo de parturição. Cogitare Enferm. v.21,n.3, p:1-8, 2016.

GRAÇA, B.C. et al. Uso da auriculoterapia no controle da lombalgia, ansiedade e estresse de profissionais do sistema penitenciário. Sociedade Brasileira para o Estudo da Dor. BrJP. São Paulo, v.3, n. 2, p:142-6, 2020.

GUIMARÃES, A.M.V. et al. Transtornos de ansiedade: um estudo de prevalência sobre as fobias específicas e a importância da ajuda psicológica. Seminário [Internet]. v.3, n.1, p:115-28, 2015. Disponível em:

https://periodicos.set.edu.br/index.php/fitsbiosaude/article/view/2611/1497. Acesso em; 11

Nov. 2020

HALL, H.G.; GRIFFITHS, D.L.; MCKENNA L.G. The use of complementary and alternative medicine by pregnant women: A literature review. Rev Midwifery.v.27, n.6, p: 817-824. 2011.

JONGSMA, H.E. et al. Incidência tratada de transtornos psicóticos no estudo multinacional EU-GEI. JAMA Psychiatry. v.75 n.1,p:36-46. 2018. Disponível em:

https://jamanetwork.com/journals/jamapsychiatry/article-abstract/2664479. Acesso em: dez. 2020.

KUREBAYASHI, L.F.S. et al. Auriculoterapia para redução de ansiedade e dor em profissionais de enfermagem: ensaio clínico randomizado. Rev. Latino-Am. Enfermagem. v.25, n.2843, p:1-9, 2017. Disponível em: https://www.scielo.br/scielo.php?pid=S0104$11692017000100320 \&$ script=sci_arttext\&tlng=pt. Acesso em: jan.2021

LEÃO, A.M. et al. Prevalência e Fatores Associados à Depressão e Ansiedade entre Estudantes Universitários da Área da Saúde de um Grande Centro Urbano do Nordeste do Brasil. Revista Brasileira de Educação Médica.v.42, n.4, p:55 - 65, 2018. 


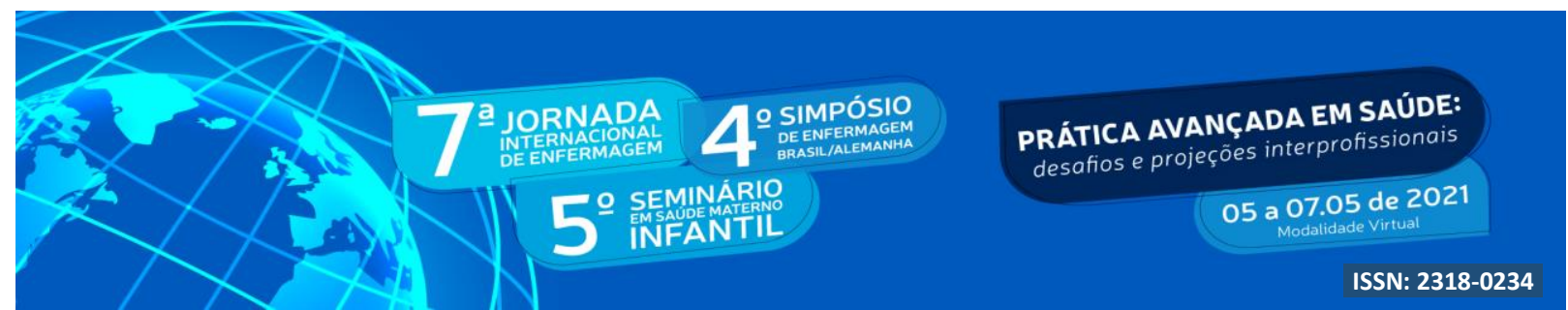

MARCHI, K.C. et al. Ansiedade e consumo de ansiolíticos entre estudantes de enfermagem de uma universidade pública. Rev. Eletr. Enf. v. 15, n.3, p:731-9, 2013. Disponível: https://revistas.ufg.br/fen/article/view/18924. Acesso em: dez.2020.

MASCARENHAS, V.H.A. et al. Evidências científicas sobre métodos não farmacológicos para alívio da dor do parto. Acta. Paul.Enferm. v.32, n.3, p:350-7, 2019.

MAFETONI, R.R. et al. Efetividade da auriculoterapia sobre a ansiedade no trabalho de parto: ensaio clínico randomizado. Rev. Latino-Am. Enfermagem. v.26, n. 3030,p:1-9, 2018. Disponível em: https://www.scielo.br/pdf/rlae/v26/pt_0104-1169-rlae-26-e3030.pdf. Acesso em: dez.2020.

MOURA, C.C. et al. Auriculoterapia efeito sobre a ansiedade. Rev.cubana de enfermeira. v. 30, n.2, 2015. Disponível em: http://www.revenfermeria.sld.cu/index.php/enf/article/view/596/90. Acesso em: dez. 2020.

ORGANIZAÇÃO MUNDIAL DE SAÚDE - OMS. Depression and other common mental disorders: global health estimates[Internet]. Geneva: WHO.p:1-24, 2017. Disponível em: http://apps.who.int/iris/bitstream/10665/254610/1/WHO-MSD-MER-2017.2-eng.pdf

PRADO, J.M.;KUREBAYASHI, L.F.S.; SILVA, M.J.P. Eficácia da auriculoterapia na redução de ansiedade em estudantes de enfermagem. Rev. Esc. Enferm.USP. v.46, n.5, p:1200-120, 2012.

SHONHOFEN, F.L. et al. Transtorno de ansiedade generalizada entre estudantes de cursos de pré-vestibular. J. Bras. Psiquiatr. v. 69, n.3, p:179-86, 2020.

SILVA, H.L. et al. Efeitos da auriculoterapia na ansiedade de gestantes no pré-natal de baixo risco. Acta Paul Enferm. v.33, n. APE20190016

p:1-8, 2020. Disponível em: https://www.scielo.br/scielo.php?pid=S010321002020000100444\&script=sci_abstract\&tlng=pt. Acesso em: dez.2020.

SILVA, R.M. et al. Uso de práticas integrativas e complementares por doulas em maternidades de Fortaleza (CE) e Campinas (SP). Saúde Soc. v.25, n.1, p.108-120, 2016. Disponível em: https://www.scielo.br/pdf/sausoc/v25n1/1984-0470-sausoc-25-01-00108.pdf. Acesso em: dez.2020.

SINTJAGO, E.M. et al. La auriculopuntura disminuye los niveles de ansiedad en adultos chilenos con malnutrición por exceso. Univ. Salud. v.20, n.3, p:304-311, 2018. Disponível em: http://dx.doi.org/10.22267/rus.182003.129. Acesso em: dez.2020.

SOUZA, M.P. Tratado de Auriculoterapia. Brasília: Novo Horizonte. pag. 3582012. 